\title{
Initial Phases of DNA Rehydration by NMR and Sorption Isotherm
}

\author{
H. HARAŃCZYK ${ }^{a, *}$, J. CZAK ${ }^{a}$, P. NOWAK ${ }^{a}$ AND J. NiziǪ ${ }^{b}$ \\ ${ }^{a}$ Institute of Physics, Jagiellonian University, Reymonta 4, 30-059 Cracow, Poland \\ ${ }^{b}$ Department of Physics and Applied Computer Sciences \\ AGH University of Science and Technology, Cracow, Poland
}

\begin{abstract}
The initial stages of rehydration of salmon sperm deoxyribonucleic acid (DNA) lyophilizates were observed using hydration kinetics, sorption isotherm, and high power proton relaxometry (at $30 \mathrm{MHz}$ ). The hydration kinetics reveals (i) a very tightly bound water not removed by incubation over silica gel $\left(A_{0}^{\mathrm{h}}=0.057 \pm 0.010\right)$, (ii) a tightly bound water [saturating at $A_{1}^{\mathrm{h}}=0.149 \pm 0.007$, hydration time $t_{1}^{\mathrm{h}}=(0.27 \pm 0.08) \mathrm{h}$ ], a tightly bound water (iii) [saturating at $A_{2}^{\mathrm{h}}=0.694 \pm 0.039$, with the hydration time $t_{2}^{\mathrm{h}}=(9.8 \pm 3.2) \mathrm{h}$ ], and (iv) a loosely bound water fraction for the samples hydrated at $p / p_{0} \geq 76 \%$ [with the hydration time $t_{3}^{\mathrm{h}}=(44 \pm 14) \mathrm{h}$, and the contribution progressively increasing with the air humidity]. For the hydration at $p / p_{0}=100 \%$, after $t_{0}=(244 \pm 22) \mathrm{h}$ of incubation the swelling process begins. The amount of additional water uptake at swelling depended on the macrostructure of the sample. Sorption isotherm is sigmoidal in form and is fitted well by the Dent model with the mass of water saturating primary binding sites $\Delta M / m_{0}=0.114$. Proton free induction decay is a superposition of the immobilized proton signal (Gaussian, with $T_{2 S}^{*} \approx 20 \mu \mathrm{s}$ ) and two liquid signal components coming from tightly bound $\left(T_{2 L_{1}}^{*} \approx 100 \mu \mathrm{s}\right.$, with the mass saturating at $\left.\Delta m / m_{0}=0.111 \pm 0.044\right)$ and loosely bound water fraction (with the amplitude proportional to the mass of water added).
\end{abstract}

PACS numbers: 82.56.Na

\section{Introduction}

For the last two decades an interest increased in developing materials which exhibit nonlinear optical properties intended for information storage or for optical signal processing. The deoxyribonucleic acid (DNA), in which charge migration takes place, is a subject of interest for its physical optical and electromagnetic properties, for potential applications in photonics and electronics [1-3]. In addition, DNA chain interact with various molecules through non-binding interactions, groove binding and intercalation, and modify their properties. This effect can be used for effective combining of already known chromophores with DNA to obtain a new materials of tailored overall properties. One of important factors, vital for understanding the properties of such a material, is good understanding of water uptake from the environment. Water content can be responsible either for optical or electronic properties.

Although the hydration properties of DNA molecule were investigated using various methods (IR [4], differential scanning calorimetry (DSC) [5], etc.), the effect of water on DNA native structure formation at the initial stages of rehydration of dry DNA molecule is still not explained.

Thus, we studied a number and distribution of water binding sites, sequence and kinetics of their saturation,

\footnotetext{
* corresponding author; e-mail: hubert.haranczyk@uj.edu.pl
}

and the formation of tightly and loosely bound water fractions at rehydration process of salmon sperm DNA, to understand the molecular mechanism of structural changes during rehydration from the anhydrous state. Taking advantage of NMR experiments, we examined the presence of water fraction "sealed" in lyophilizate structure and the effect of the sample swelling occurring for hydratation from $p / p_{0}=100 \%$.

\section{Materials and methods}

DNA sodium salts, extracted from salmon milt and roe were provided by Chitose Institute of Science and Technology, Japan. The molecular weight measured $\mathrm{MW}=10^{6} \mathrm{Da}$ (or 2000 base pairs). The salmon sperm DNA lyophilizates in air-dry form are hydrated to $\Delta m / m_{0}=0.146 \pm 0.006$, where $m_{0}$ is the dry mass of the sample, and $\Delta m$ is mass of water taken up by DNA (determined gravimetrically).

Before the hydration experiments the samples were incubated for $250 \mathrm{~h}$ over silica gel (at relative humidity, $p / p_{0}=0 \%$ ), dehydrating to the hydration level $\Delta m / m_{0}=0.052 \pm 0.006$. The dehydration kinetics was single exponential with the dehydration time constant $t_{1}^{\mathrm{d}}=(5.29 \pm 0.28) \mathrm{h}$.

The hydration courses were performed from the gaseous phase with the controlled humidity, at room temperature, over the surface of $\mathrm{H}_{3} \mathrm{PO}_{4}\left(p / p_{0}=9 \%\right)$, over the surfaces of saturated solutions of $\mathrm{KC}_{2} \mathrm{H}_{3} \mathrm{O}_{2}\left(p / p_{0}=\right.$ $23 \%), \mathrm{CaCl}_{2}\left(p / p_{0}=32 \%\right), \mathrm{K}_{2} \mathrm{CO}_{3}(44 \%), \mathrm{Na}_{2} \mathrm{Cr}_{2} \mathrm{O}_{7}$ 
$\left(p / p_{0}=52 \%\right), \mathrm{NH}_{4} \mathrm{NO}_{3}(63 \%), \mathrm{Na}_{2} \mathrm{~S}_{2} \mathrm{O}_{3}(76 \%), \mathrm{K}_{2} \mathrm{CrO}_{3}$ (88\%), $\mathrm{Na}_{2} \mathrm{SO}_{4}(93 \%), \mathrm{K}_{2} \mathrm{SO}_{4}(97 \%)$, and over a water surface $\left(p / p_{0}=100 \%\right)$.

After completing the hydration courses, the dry mass of the samples was determined after heating at $150^{\circ} \mathrm{C}$. After heating for $1 \mathrm{~h}$, the sample was weighted, and heating was continued. No further decrease in sample mass was recorded. The thermal decomposition of DNA starts at $200^{\circ} \mathrm{C}[5]$.

Proton free induction decays (FIDs) were recorded on WNS HB-65 high power relaxometer (Waterloo NMR Spectrometers, St. Agatha, Ontario, Canada). The resonance frequency was $30 \mathrm{MHz}$ (at $\left.B_{0}=0.7 \mathrm{~T}\right)$; the transmitter power was $400 \mathrm{~W}$; the pulse length $\pi / 2=1.5 \mu \mathrm{s}$. Data were acquired using Compuscope 2000 card in an IBM clone computer, controlling the spectrometer, and averaged over 2000 accumulations. Repetition time was $2.003 \mathrm{~s}$. The measurements were performed at room temperature.

The data were analyzed using the one-dimensional, FID analyzing procedure of the two-dimensional (in time domain) NMR signal-analyzing program CracSpin written at the Jagiellonian University, Cracow [6]. The swelling was fitted using program Origin, and the threshold function for swelling triggering was modeled by $\frac{1}{2}\left\{1+\tanh \left[\alpha\left(t-t_{0}\right)\right]\right\}$ function for high values of the parameter $\alpha$.

\section{Results}

\subsection{Hydration kinetics}

The hydration courses for salmon sperm DNA lyophilizates performed from the gaseous phase are fitted well by single exponential function for $p / p_{0} \leq 32 \%$ (see Fig. 1)

$$
\Delta m(t) / m_{0}=A_{0}^{\mathrm{h}}+A_{1}^{\mathrm{h}}\left[1-\exp \left(-t / t_{1}^{\mathrm{h}}\right)\right],
$$

where $\Delta m / m_{0}$ is the relative mass increase, $A_{0}^{\mathrm{h}}$ is the saturation level for very tightly bound water fraction (i) level not removed by incubation over silica gel $\left(p / p_{0}=0 \%\right)$, $A_{1}^{\mathrm{h}}$ is the saturation level for the tightly bound water fraction (ii), and $t_{1}^{\mathrm{h}}$ is the corresponding hydration time constant. The averaged over the all target humidities, $A_{0}^{\mathrm{h}}$ equals $0.057 \pm 0.010$. For tightly bound water component $A_{1}^{\mathrm{h}}=0.149 \pm 0.007$, whereas the hydration time $t_{1}^{\mathrm{h}}=(0.27 \pm 0.08) \mathrm{h}$.

For the hydration from $p / p_{0}$ between $32 \%$ and $63 \%$ the hydration course is better fitted by the two-exponential function (Fig. 1)

$$
\begin{aligned}
& \Delta m(t) / m_{0}=A_{0}^{\mathrm{h}}+A_{1}^{\mathrm{h}}\left[1-\exp \left(-t / t_{1}^{\mathrm{h}}\right)\right] \\
& \quad+A_{2}^{\mathrm{h}}\left[1-\exp \left(-t / t_{2}^{\mathrm{h}}\right)\right],
\end{aligned}
$$

where $A_{2}^{\mathrm{h}}=0.694 \pm 0.039$ is the saturation level for the tightly bound water fraction (iii), and $t_{2}^{\mathrm{h}}=(9.8 \pm 3.2) \mathrm{h}$ is the corresponding hydration time constant.

For the samples hydrated from $p / p_{0} \geq 76 \%$ the third exponential component of the hydration course is recorded (see Fig. 1)

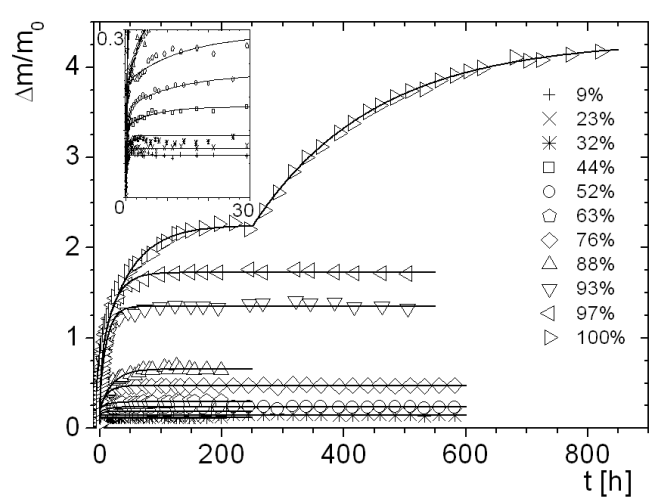

Fig. 1. Hydration kinetics of salmon sperm DNA lyophilizates performed from the gaseous phase (the target humidities are marked in the inset). The errors are within the plot symbols.

$$
\begin{aligned}
& \Delta m(t) / m_{0}=A_{0}^{\mathrm{h}}+A_{1}^{\mathrm{h}}\left[1-\exp \left(-t / t_{1}^{\mathrm{h}}\right)\right] \\
& \quad+A_{2}^{\mathrm{h}}\left[1-\exp \left(-t / t_{2}^{\mathrm{h}}\right)\right]+A_{3}^{\mathrm{h}}\left[1-\exp \left(-t / t_{3}^{\mathrm{h}}\right)\right],
\end{aligned}
$$

where $A_{3}^{\mathrm{h}}$ is the saturation level for the loosely bound water fraction (iv), and $t_{3}^{\mathrm{h}}$ is the corresponding hydration time constant. The contribution of the loosely bound water component progressively increased with the air humidity, and the hydration time equals and $t_{3}^{\mathrm{h}}=$ $(44 \pm 14)$ h.

\subsection{Swelling}

The salmon sperm DNA lyophilizate rehydrated from $p / p_{0}=100 \%$ shows the additional hydration process starting at $t_{0}=(244 \pm 22) \mathrm{h}$. For several samples hydrated the amplitude of swelling varied depending on the macroscopic form of the lyophilizate fibers, however, the time constant for triggering of the swelling process, $t_{0}$, was not changed. The swelling process was well fitted using the two-step function

$$
\begin{aligned}
& \Delta m(t) / m_{0}=A_{0}^{\mathrm{h}}+A_{1}^{\mathrm{h}}\left[1-\exp \left(-t / t_{1}^{\mathrm{h}}\right)\right] \\
& +A_{2}^{\mathrm{h}}\left[1-\exp \left(-t / t_{2}^{\mathrm{h}}\right)\right]+A_{3}^{\mathrm{h}}\left[1-\exp \left(-t / t_{3}^{\mathrm{h}}\right)\right] \\
& +\left\{\begin{array}{c}
0 \text { for } t<t_{0}, \\
A_{4}^{\mathrm{h}}\left[1-\exp \left(-t / t_{4}^{\mathrm{h}}\right)\right] \text { for } t>t_{0},
\end{array}\right.
\end{aligned}
$$

where $A_{4}^{\mathrm{h}}$ is the swelling amplitude, and $t_{4}^{\mathrm{h}}$ is the swelling time. Swelling process strongly depended on the sample and varied in amplitude between $0.215 \pm 0.065$ and $2.014 \pm 0.023$ and in the swelling time constant $t_{0}$ between $(25 \pm 23) \mathrm{h}$ and $(202 \pm 5) \mathrm{h}$, respectively.

\subsection{Sorption isotherm}

The total saturation hydration level, $C^{\mathrm{h}}$, was calculated as

$$
C^{\mathrm{h}}=\sum_{i=0}^{n} A_{i},
$$

where $n$ equals 1,2 or 3 depending on hydration level 
(see Eq. (1a), (1b) or (1c)), and subsequently was taken for the construction of sorption isotherm.

For salmon sperm DNA lyophilizates the sorption isotherm is sigmoidal in form (Fig. 2), which usually is fitted by Dent [7, 8] and/or by BET [9] model. Both models distinguish two types of water binding sites, namely, (i) "primary" water binding sites (directly to the adsorbent surfaces); and (ii) "secondary", usually weaker, water binding sites (to the surfaces binding sites with small affinity, to the primary bound water molecules, or to the previous water layers). The difference is that BET model takes a fixed value of the ratio of the number of binding sites covered by $n$ water molecules to that covered by $n-1$ water molecules, $b=S_{n} /\left.S_{n-1}\right|_{h=1}=1$ (which is an artificial assumption), whereas the Dent model allows varying of this ratio between 0 and 1 (better modeling droplet formation).

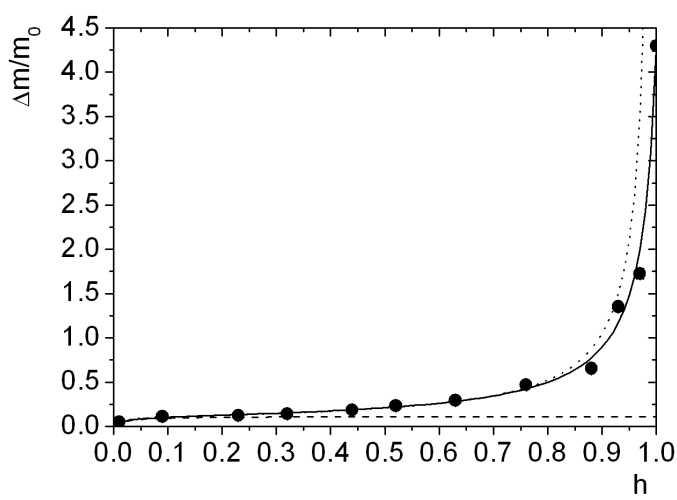

Fig. 2. The sorption isotherm for salmon sperm DNA lyophilizates (solid line - Dent model, dashed line BET model, dotted line - monolayer coverage calculated from Dent model). The values of $h\left(=p / p_{0}\right)$ represent the relative humidity and the values of relative mass increase, $\Delta m / m_{0}$, are taken as the saturation values $C^{\mathrm{h}}$ from Eq. (2).

The sorption isotherm for both models is described by

$$
C^{\mathrm{h}}(h)=\frac{\Delta M}{m_{0}} \frac{b_{1} h}{(1-b h)\left(1+b_{1} h-b h\right)},
$$

where $h$ is relative humidity $p / p_{0}$, expressed in absolute units, $\Delta M / m_{0}$ is the mass fraction of water saturating primary binding sites. At $h=1$ the contribution of empty primary binding sites, $S_{0}$, is expressed through the reciprocal of $b_{1}$ as $S_{0} /\left.S_{1}\right|_{h=1}=1 / b_{1}$.

To test the relevance of sorption model the sorption isotherm is usually presented in parabolic form (see Fig. 3). The parabolic form of Dent isotherm is described by

$$
\frac{h}{\Delta m / m_{0}}=A+B h-C h^{2},
$$

where parameters $\frac{\Delta M}{m_{0}}, b, b_{1}$ are connected with $A, B, C$ by the formulae

$$
b=\frac{\sqrt{B^{2}+4 A C}-B}{2 A}, \quad b_{1}=\frac{B}{A}+2 b,
$$

$$
\frac{\Delta M}{m_{0}}=\frac{1}{A b_{1}} .
$$

The parabolic form of BET isotherm is described by

$$
\frac{h}{\Delta m / m_{0}}=A+B h-(A+B) h^{2} \text {. }
$$

For salmon sperm DNA lyophilizates the sorption isotherm is much better described by Dent model (see Fig. 3). The mass of water saturating primary water binding sites equals $\Delta M / m_{0}=0.114$. The contribution from empty binding sites at $h=1$ is given by $1 / b_{1}=2.95 \%$ (expressed as percentage). The model parameter $b$, indicating the applicability of Dent model, is equal to 0.965 .

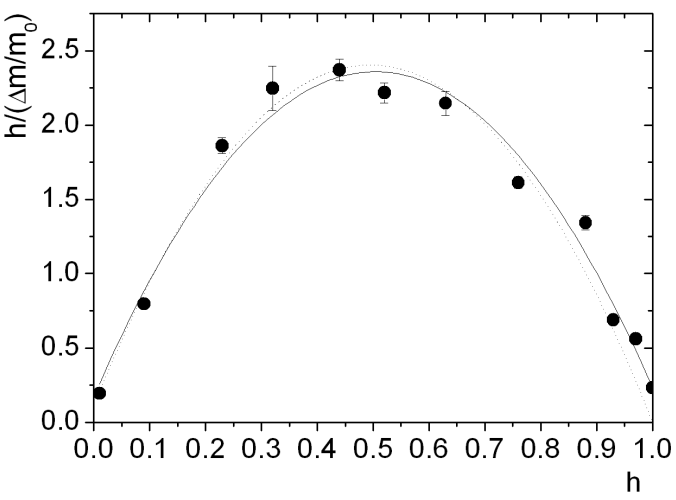

Fig. 3. Parabolic form of Dent and BET model (closed circles $=$ experimental data, solid line $=$ fitted Dent model, dotted line $=$ BET model).

\subsection{Proton free induction decays}

The free induction decays for protons of the salmon sperm DNA lyophilizates, at lower hydration levels $\left(\Delta m / m_{0} \leq 0.152\right)$, are well fitted by the superposition of one Gaussian component, with the amplitude $S$, coming from immobilized protons, and one exponential component, $L_{1}$, coming from water tightly bound on the surfaces of the membrane, whereas for higher hydration levels loosely bound water component, $L_{2}$, appears

$$
\begin{aligned}
& F I D(t)=S \exp \left(-\left(\frac{t}{T_{2 S}^{*}}\right)^{2}\right)+L_{1} \exp \left(-\frac{t}{T_{2 L_{1}}^{*}}\right) \\
& +L_{2} \exp \left(-\frac{t}{T_{2 L_{2}}^{*}}\right),
\end{aligned}
$$

where $T_{2 S}^{*}$ is the proton spin-spin relaxation time of solid component taken as the 1/e-value of Gaussian solid signal, and $T_{2 L_{1}}^{*}$ and $T_{2 L_{2}}^{*}$ are the relaxation times of proton liquid fractions $L_{1}$ and $L_{2}$, respectively. A typical FID is shown in Fig. 4.

For very high hydration levels $\left(\Delta m / m_{0} \geq 0.950\right)$ fitting procedure fails in fitting of relatively small tightly bound water signal, so, solely loosely bound water component is detected.

Figure 5 shows the rehydration dependence of the proton relaxation times for the components of FID signal 


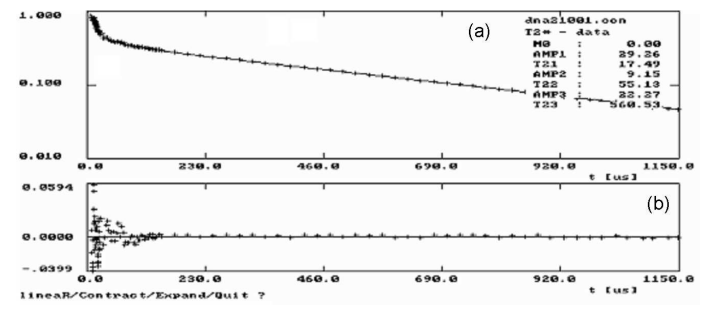

Fig. 4. (a) Proton free induction decay recorded for salmon sperm DNA lyophilizates at $30 \mathrm{MHz}$; the pulse length $\pi / 2=1.4 \mu \mathrm{s}$. The relative mass increase was $\Delta m / m_{0}=0.262$. $Y$-axis shows the normalized signal amplitude. The solid line represents a least squares fit of Eq. (6) to the data. (b) The residual function calculated as the difference between the fitted and recorded values of the FID signal, which for any recorded point does not exceed $3.3 \%$.

of salmon sperm DNA lyophilizates. The solid signal was Gaussian in form, the characteristic for many micro-heterogeneous dry biological systems "beat" pattern was not seen even in residual function $[10,11]$.

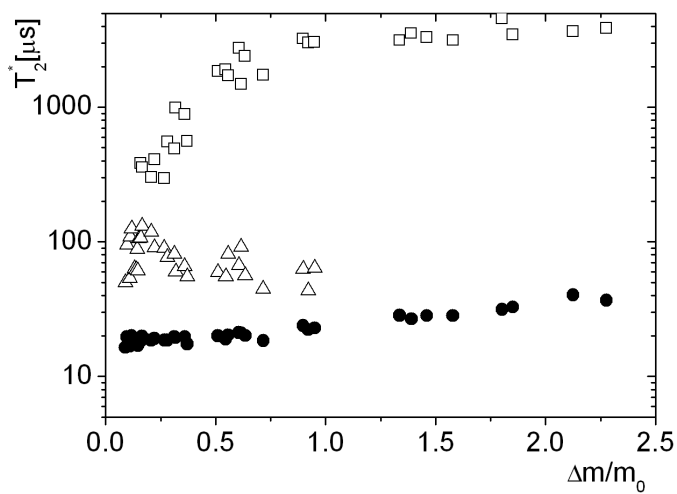

Fig. 5. The hydration dependence of proton FID relaxation times for salmon sperm DNA lyophilizates. Solid Gaussian, $S$, component - closed circles; tightly bound water, $L_{1}$, component $-\times$, and loosely bound water, $L_{2}$, component - open circles.

For the hydration levels $\Delta m / m_{0} \leq 0.950$ the spinspin relaxation time for solid component was equal to $T_{\mathrm{S}, 0}^{*} \approx 19 \mu \mathrm{s}$, whereas for higher hydration levels its value increases, which may be caused by numeric reasons, because the short $L_{1}$ component is no longer fitted. The value of the spin-spin relaxation time for solid is close to that value for the solid matrices of several dry microheterogeneous biological systems (e.g. model DGDG membranes [12], photosynthetic membranes [13], bark and bast [14], wheat seed [15], lichen thallus [11, 16-19], dentine and dental enamel [20]). Moreover, similar values of spin-spin relaxation time are recorded for dry solid polymer matrices [21]. Thus, we assigned the Gaussian component, $S_{0}$, to solid matrix of the salmon sperm lyophilizate, and used this amplitude as a unit to scale the amplitudes of the other signal components.
For the mobile proton fraction $L_{1}$, the value of the decay time does not depend much on the hydration level and equals $T_{2 L_{1}}^{*} \approx 80 \mu \mathrm{s}$. Proton FID time for the $L_{1}$ fraction resembles that for the immobilized (tightly bound) water signal of photosynthetic membranes [13], bark and bast [14], wheat seed [15], lichen thallus [11, 16-19], dentine and dental enamel [20], and of the controlled pore glasses [22].

The relaxation time $T_{2 L_{2}}^{*}$ for longest mobile signal component increases with the increasing hydration level (Fig. 5), suggesting that this component is an average of some proton subsystems being in fast exchange regime [23]. For higher hydration levels the $T_{2 L_{2}}^{*}$ values are of the order of $\approx 3 \mathrm{~ms}$. Thus, the $L_{2}$ component is either a loosely bound water fraction, or free water. The spin-spin relaxation times $T_{2 L_{2}}^{*}$ measured in FID experiment are shortened by $B_{0}$ inhomogeneities [24]:

$$
\frac{1}{T_{2}^{*}}=\frac{1}{T_{2}}+\frac{\gamma \Delta B_{0}}{2}
$$

where $T_{2}$ is spin-spin relaxation time, $\gamma$ is gyromagnetic ratio, and $\Delta B_{0}$ is a change of magnetic field $B_{0}$ within the sample. The Gaussian fits performed in frequency domain yield for $L_{2}$ line the halfwidths equal $\Delta \omega=530 \mathrm{~Hz}$ $\left(\Delta B_{0}=0.012 \mathrm{mT}\right)$.

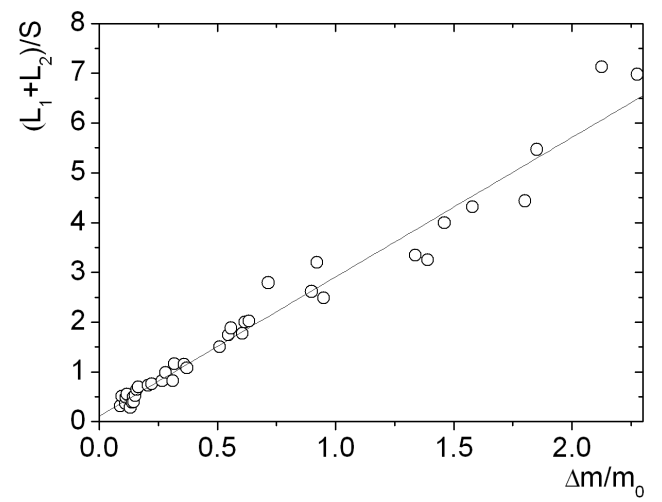

Fig. 6. The $\left(L_{1}+L_{2}\right) / S$ hydration dependence for rehydrated salmon sperm DNA lyophilizates. The solid line was calculated from $\left(L_{1}+L_{2}\right) / S=(2.80 \pm$ $0.09) \Delta m / m_{0}+(0.111 \pm 0.081)$.

Although the solid and short exponential components in the FID experiment are not changed by $\Delta B_{0}$ as compared to those measured by spin echo method [25], the measured $T_{2 L_{2}}^{*}$ are significantly shortened.

The total mobile proton signal expressed in units of solid is linear with the increased hydration level and is well fitted by the dependence (Fig. 6)

$$
\begin{aligned}
& \left(L_{1}+L_{2}\right) / S=(2.80 \pm 0.09) \Delta m / m_{0} \\
& \quad+(0.111 \pm 0.081) .
\end{aligned}
$$

The constant term in Eq. (10) may reflect the signal coming from water "sealed" in pores of solid structure $[13,14]$. However, as compared to some other dry systems [13] its contribution is very small, if any. The linear 


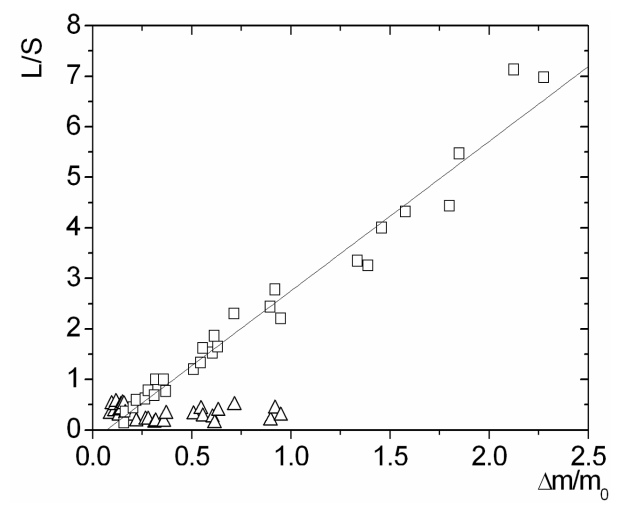

Fig. 7. The $L_{1} / S$ and $L_{2} / S$ hydration dependence (crosses and closed circles, respectively) for rehydrated salmon sperm DNA lyophilizates. The solid line was calculated from $L_{2} / S=(2.96 \pm 0.11) \Delta m / m_{0}+$ $(-0.21 \pm 0.11)$. The amplitude of $L_{1}$ component remained constant for the whole hydration range, and $L_{1} / S=0.33 \pm 0.13$.

form of the dependence suggests the absence of water soluble solid fraction $[14,19]$.

The hydration dependence of loosely bound water fraction signal is expressed by the linear function (Fig. 7)

$L_{2} / S=(2.96 \pm 0.11) \Delta m / m_{0}+(-0.21 \pm 0.11)$.

The amplitude of $L_{1}$ component does not change in the investigated hydration range $\Delta m / m_{0} \geq 0.089$ (see Fig. 7), and is equal to $L_{1} / S=0.33 \pm 0.13$. The value of the slope is smaller compared to those for thallus of Usnea antarctica (3.6 [18]), and for mature (3.9) and developing (3.0) photosynthetic membranes [26], suggesting the decreased contribution of paramagnetic ions in the system. The slope of the $L_{2} / S$ dependence may be used to calculate the contribution of tightly bound water fraction in salmon sperm DNA as equal to $\Delta m / m_{0}=0.111 \pm 0.044$, which is equal to water fraction saturating primary water binding sites, as calculated from sorption isotherm.

\subsection{NMR sorption isotherm}

The hydration dependence of total liquid signal may be used to construct the NMR-isotherm, with the same sorption parameters $\left(\Delta m / m_{0}, b\right.$, and $\left.b_{1}\right)$ obtained from gravimetry $[11,18,19,26]$. Only the constant water component, $A$, "sealed" in DNA lyophilizate structures, and the proportionality coefficient $k$, scaling NMR signal in units of water mass, should be fitted. The proportionality coefficient depends on the ratio of the screening effect of the liquid signal to that on the solid signal caused by paramagnetic ions present in the system. This method allows the combination of the classic sorption isotherm with NMR hydration data.

The normalized amplitude of NMR total liquid signal expressed as a function of $h$ was fitted to the function

$$
\frac{L_{1}+L_{2}}{S_{0}}(h)=A+k \frac{\Delta M}{m_{0}} \frac{b_{1} h}{(1-b h)\left(1+b_{1} h-b h\right)} .
$$

The solid line was calculated from Eq. (10) with best-fit

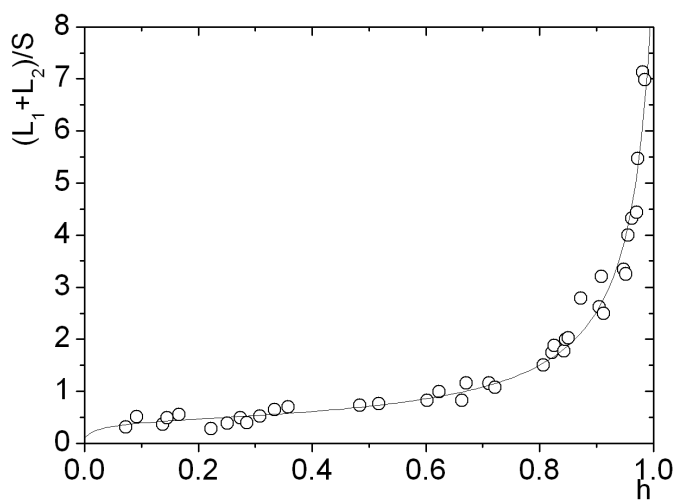

Fig. 8. Sorption isotherm fitted to total FID proton signal for water hydrating salmon sperm DNA lyophilizate. Closed squares - experimental data, solid line - Dent model Eq. (4).

parameters $k=2.800 \pm 0.090$ and $A=0.111 \pm 0.082$. The contribution of "sealed" water fraction equals $\Delta m / m_{0}=$ $0.038 \pm 0.028$ which does not exceed much the experimental error, suggesting the absence of water fraction "sealed" in the structure of DNA lyophilizate.

\section{Discussion}

The swelling of rehydrated salmon sperm DNA lyophilizate may be described using two models. First, the outer layer of the fibers is more solid hindering the hydration process kinetics. The outer layer is hydrated at first leading finally to increase water permeability to the core of the fiber. The observed different swelling process effectiveness may be explained in terms of different thickness of outer layer and of different volume of fiber core. The second model suggests that the fiber is homogeneous and hydrating water penetrates its whole volume. With the increase of the fiber hydrating layer, the fiber is destabilized and additional process of decomposed structure starts. We are in favor of the second model. X-ray data show that in solid form DNA arranges in fibers, forming hexagonal lattice with the constant $a$ increasing with the humidity [27, 28]. For the highest humidities the DNA molecules dissolves in aqueous medium [29], which was also observed by us for the samples with high mass of water taken up at swelling.

Either hydration kinetics or NMR-sorption isotherm suggests the absence of water fraction "sealed" in dry lyophilizate of salmon sperm DNA. This means that the structure of DNA lyophilizate is uniform, and is an additional argument to the proposed second model of swelling process.

\section{References}

[1] J. Grote, J. Hagen, J. Zetts, R. Nelson, D. Diggs, M. Stone, P. Yaney, E. Heckman, C. Zhang, W. Steier, A. Jen, L. Dalton, N. Ogata, M. Curley, S. Clarson, F. Hopkins, J. Phys. Chem. B 108, 8584 (2004). 
[2] H. Fink, H. Schmid, E. Ermantraut, T. Schulz, J. Opt. Soc. Am. A 14, 2168 (1997).

[3] B. Singh, M. Sariciftci, J. Grote, F.K. Hopkins, J. Appl. Phys. 100, 024514 (2006).

[4] M. Falk, K.A. Hartman, Jr., R.C. Lord, J. Am. Chem. Soc. 85, 387 (1963).

[5] S.L. Lee, P.G. Debenedetti, J.R. Errington, B.A. Pethica, D.J. Moore, J. Phys. Chem. B 108, 3098 (2004).

[6] W. Weglarz, H. Harańczyk, J. Phys. D, Appl. Phys. 33, 1909 (2000).

[7] R.W. Dent, Textile Res. J. 47, 145 (1977).

[8] A.J. Hailwood, S. Horrobin, Trans. Faraday Soc. B 42, 84 (1946).

[9] S. Brunauer, P.H. Emmett, E. Teller, J. Am. Chem. Soc. 60, 309 (1938).

[10] W. Derbyshire, M. Van, Den Bosch, D. Van Dusschoten, W. MacNaughtan, I.A. Farhat, M.A. Hemminga, J.R. Mitchell, J. Magn. Res. 168, 278 (2004).

[11] H. Harańczyk, M. Bacior, M.A. Olech, Antarctic Sci. 20, 527 (2008).

[12] H. Harańczyk, M. Bacior, J. Jamróz, M. Jemioła-Rzemińska, K. Strzałka, Acta Phys. Polon. A $\mathbf{1 1 5}$ 521 (2009).

[13] H. Harańczyk, A. Leja, K. Strzałka, Acta Phys. Polon. A 109, 389 (2006).

[14] H. Harańczyk, W.P. Węglarz, S. Sojka, Holzforschung 53, 299 (1999).

[15] H. Harańczyk, K. Strzałka, G. Jasiński, K. Mosna-Bojarska, Coll. Surf. A 115, 47 (1996).

[16] H. Harańczyk, S. Gaździński, M.A. Olech, New Phytologist 138, 191 (1998).
[17] H. Harańczyk, S. Gaździński, M. Olech, Freezing protection mechanism in Cladonia mitis as observed by proton magnetic relaxation. in: New Aspects in Cryptogamic Research, Contribution in Honour of Ludger Kappen. Bibl. Lichenol. 75, 265 (2000).

[18] H. Harańczyk, A. Pietrzyk, A. Leja, M.A. Olech, Acta Phys. Polon. A 109, 411 (2006).

[19] H. Harańczyk, M. Bacior, P. Jastrzębska, M.A. Olech, Acta Phys. Polon. A 115, 516 (2009).

[20] N. Funduk, G. Lahajnar, L. Miljković, S. Skočajić, D.W. Kydon, L.J. Schreiner, M.M. Pintar, Zobozdrav. Vestn. 41 (Suppl. 1), 139 (1986).

[21] W. Węglarz, H. Peemoeller, A. Rudin, Polym. Sci. B (Polymer Physics) 38, 2487 (2000).

[22] H. Harańczyk, K.G. Soga, R.J. Rumm, M.M. Pintar, Magn. Res. Imag. 9, 723 (1991).

[23] J.R. Zimmerman, W.E. Brittin, J. Phys. Chem. 61, 1328 (1957)

[24] A. Timur, J. Petroleum Technol. 21, 775 (1969).

[25] T.J. Wydrzynski, S.B. Marks, P.G. Schmidt, H.S. Govindjee, Gutowsky, Biochemistry 17, 2155 (1978).

[26] H. Harańczyk, A. Leja, M. Jemioła-Rzemińska, K. Strzałka, Acta Phys. Polon. A 115, 526 (2009).

[27] A. North, A. Rich, Nature 191, 1242 (1961).

[28] R. Greenall, C. Nave, W. Fuller, J. Mol. Biol. 305, 669 (2001).

[29] S. Zimmerman, B. Pheiffer, Proc. Natl. Acad. Sci. USA 76, 2703 (1979) 